Pacific Journal of Mathematics

THE CLASS OF $(p, q)$-BIHARMONIC FUNCTIONS 


\title{
THE CLASS OF $(p, q)$-BIHARMONIC FUNCTIONS
}

\section{Leo Sario and Cecilia Wang}

\begin{abstract}
Our main interest in this paper is with the $(p, q)$-biharmonic boundary value problem, which takes the following form: Given continuous functions $\phi$ and $\Psi$ on Wiener's or Royden's $p$-and $q$-harmonic boundaries $\alpha$ and $\beta$ respectively, find a function $u$ satisfying $(\Delta+q)(\Delta+p) u=0$ and

$$
u|\alpha=\varphi, \quad u| \beta=\Psi .
$$

We shall solve this problem by what we call the $(p, q)$ biharmonic projection.
\end{abstract}

In $\S 1$ we give some preliminary results. The $(p, q)$-biharmonic projection is introduced in $\S 2$ for various classes of functions, and in $\S 4$ for suitably restricted Riemannian manifolds. In $\S 3$ we characterize classes of manifolds with respect to significant subclasses of $(p, q)$ quasiharmonic functions by means of the $p$-harmonic Green's function and the $q$-elliptic measure on $R$. The $(p, q)$-quasiharmonic nondegeneracies of the manifold are the various conditions we impose on $R$ in $\S 4$. Finally in $\S 5$ we give some explicit results concerning certain classes of density functions.

1. On a smooth noncompact Riemannian manifold $R$ of dimension $m \geqq 2$ with a smooth metric tensor $\left(g_{i j}\right)$, the Laplace-Beltrami operator is given by

$$
\Delta \cdot=-\frac{1}{\sqrt{g}} \sum_{i=1}^{m} \frac{\partial}{\partial x^{i}} \sum_{j=1}^{m} \sqrt{g} g^{i j} \frac{\partial \cdot}{\partial x^{j}},
$$

where $x=\left(x^{1}, \cdots, x^{m}\right)$ is a local coordinate system, $g=\operatorname{det}\left(g_{i j}\right)$, and $\left(g^{i j}\right)=\left(g_{i j}\right)^{-1}$. Let $p(x)$ be a density function, that is, a nonnegative $C^{2}$ function on $R$. A $p$-harmonic function is a $C^{2}$ solution of the equation $\Delta_{p} u=0$ with

$$
\Delta_{p}=\Delta+p
$$

We call a $C^{4}$ function $(p, q)$-biharmonic if it satisfies the equation

$$
\Delta_{q} \Delta_{p} u=0,
$$

and we denote by $W_{p q}=W_{p q}(R)$ the family of $(p, q)$-biharmonic functions on $R$. An important subclass of $W_{p q}$ is the class $Q_{p q}=Q_{p q}(R)$ of $(p, q)$-quasiharmonic functions, i.e., the $C^{2}$ solutions of $\Delta_{p} u=e_{q}$, where $e_{q}$ is the $q$-elliptic measure on $R$ (see No. 2). 
Note that for $p \equiv q \equiv 0, W_{p q}$ and $Q_{p q}$ reduce to the classes $W$ and $Q$ of biharmonic and quasiharmonic functions respectively. For these classes our problem was solved in [ 5 ]-[ 7 ] and [11], which have greatly influenced our reasoning.

\section{Auxiliary results.}

2. Let $\Omega$ be a regular subregion of $R$, and $h_{1}^{p \Omega}$ the continuous function on $R$ which is $p$-harmonic on $\Omega$ and 1 on $R-\Omega$. The limit $e_{p}$ of the decreasing sequence $\left\{h_{1}^{p .}\right\}$ as $\Omega \rightarrow R$ is called the p-elliptic measure of $R$. Clearly $e_{p}$ is nonnegative and $p$-harmonic on $R$, with $0 \leqq e_{p} \leqq 1$. Explicitly, it is either identically zero or strictly positive. In particular, it is identically 1 if $p \equiv 0$. In the case $p \neq \equiv 0$, we call a Riemannian manifold $R$-parabolic if $e_{p}=0$, and $p$-hyperbolic if $e_{p}>0$. As in the case $p \equiv 0$, we shall follow the convention adopted by Royden [9] that $R$ is called 0-parabolic if and only if $R$ is parabolic.

3. The harmonic Green's function $g(x, y)$ on $R$ exists only on a hyperbolic manifold. In contrast, the $p$-harmonic Green's function $g_{p}(x, y)$ for $p \not \equiv 0$ exists on every Riemannian manifold. Thus on an arbitrary Riemannian manifold $R$, hyperbolic if $p \equiv 0$, the operator $G_{p}$ is well defined on the family of continuous functions by

$$
G_{p} f=\int_{R} g_{p}(\cdot, y) f(y) d y,
$$

with $d y$ the volume element of $R$. We are interested in the class

$$
F_{p_{1}}=\left\{f\left|G_{p}\right| f \mid<\infty\right\} \text {. }
$$

Lemma 1. Let $R$ be an arbitrary Riemannian manifold (hyperbolic if $p \equiv 0)$. If $f \in C^{\infty} \cap F_{p_{1}}$, then $\Delta_{p} G_{p} f=f$.

Proof. For every $\phi \in C_{0}^{\infty}$, we have

$$
\begin{aligned}
\int_{R} G_{p} f(x) \cdot \Delta_{p} \varphi(x) d x & =\int_{R} G_{p} \Delta_{p} \varphi(y) \cdot f(y) d y \\
& =\int_{R} \varphi(y) \cdot f(y) d y .
\end{aligned}
$$

Therefore $\Delta_{p} G_{p} f=f$ in the sense of distributions, and the lemma follows by the hypoellipticity of $\Delta_{p}$.

4. Let $M_{p_{1}}(R)$ be the class of continuous $p$-harmonizable functions for which there is a continuous $p$-superharmonic function $s_{f}$ with $s_{f} \geqq|f|$ on $R$, and $N_{p_{1}}(R)$ the potential $p$-subalgebra of $M_{p_{1}}(R)$, i.e., 
the family of functions in $M_{p_{1}}(R)$ whose $p$-harmonic part $h_{f}^{p}=\lim _{\Omega \rightarrow R} h_{f}^{p .}$ vanishes identically on $R$.

Lemma 2. Let $R$ be an arbitrary Riemannian manifold (hyperbolic if $p \equiv 0)$. If $f \in C^{\infty} \cap F_{p_{1}}$, then $G_{p} f \in N_{p_{1}}(R)$.

Proof. Set $f=f^{+}-f^{-}$with $f^{+}=f \cup 0$ and $f^{-}=-f \cup 0$. Clearly $G_{p} f^{+}$and $G_{p} f^{-}$are nonnegative and p-superharmonic on $R$. In view of $\left|G_{p} f\right| \leqq G_{p} f^{+}+G_{p} f^{-}, G_{p} f \in M_{p_{1}}(R)$.

It remains to show that $h_{G_{p} f}^{p}=0$. Let $\Omega$ be a regular subregion of $R$ and $g_{p, \Omega}(x, y)$ the $p$-harmonic Green's function on $\Omega$ with value zero on $R-\Omega$. For a parametric disk $B_{x} \subset R$ about $x \in \Omega$ with radius $\varepsilon$, the Green's formula yields

$$
\begin{aligned}
& \int_{\partial\left(\Omega-B_{x}\right)}\left\{\left[G_{p} f(y)-h_{G_{p} f}^{p \Omega}(y)\right] * d g_{p \Omega}(x, y)-g_{p \Omega}(x, y) * d\left[G_{p} f(y)-h_{G_{p} f}^{p \Omega}(y)\right]\right\} \\
= & \int_{\Omega-B_{x}} g_{p \Omega}(x, y) \Delta_{p} G_{p} f(y) d y .
\end{aligned}
$$

On letting $\varepsilon \rightarrow 0$ and then $\Omega \rightarrow R$ we obtain

$$
G_{p} f(x)=h_{G_{p} f}^{p \Omega}(x)+\int_{R} g_{p \Omega}(x, y) \Delta_{p} G_{p} f(y) d y
$$

and by Lemma 1,

$$
G_{p} f=h_{G_{p} f}^{p}+G_{p} f .
$$

Therefore $h_{G_{p} f}^{p}=0$ and consequently $G_{p} f \in N_{p_{1}}(R)$.

5. Denote by $H^{p}(R)$ the class of $p$-harmonic functions on $R$, and let $E(u)$ be the energy integral

$$
E(u)=\int_{R} d u \wedge * d u+\int_{R} p(x) u^{2}(x) d x .
$$

LEMmA 3. The energy integral is lower semicontinuous:

$$
E\left(u_{0}\right) \leqq \lim _{n \rightarrow \infty} E\left(u_{n}\right)
$$

for every sequence $\left\{u_{n}\right\}$ in $H^{p}(R)$ converging uniformly to $u_{0}$ on compacta of $R$.

Proof. For $x_{0} \in R$ and a parametric ball $B \subset R$ about $x_{0}$,

$$
u_{n}(x)=-\int_{\partial B} u_{n}(y) \frac{\partial g_{p B}(x, y)}{\partial n_{y}} d S_{y}, \quad n=0,1,2, \cdots,
$$

with $x \in B, g_{p_{B}}$ the $p$-Green's function on $B, \partial g_{p_{B}} / \partial n$ the normal deriva- 
tive of $g_{p B}$, and $d S$ the surface element of $\partial B$. Then

$$
\begin{aligned}
\frac{\partial u_{n}(x)}{\partial x^{i}} & =-\int_{\partial B} u_{n}(y) \frac{\partial}{\partial x^{i}} \frac{\partial g_{p B}(x, y)}{\partial n_{y}} d S_{y} \\
& \rightarrow-\int_{\partial B} u_{0}(y) \frac{\partial}{\partial x^{i}} \frac{\partial g_{p B}(x, y)}{\partial n_{y}} d S_{y}=\frac{\partial u_{0}(x)}{\partial x^{i}}
\end{aligned}
$$

as $n \rightarrow \infty$. Therefore $\partial u_{n}(x) / \partial x^{i} \rightarrow \partial u_{0}(x) / \partial x^{i}$ uniformly on every parametric ball as $n \rightarrow \infty$. The uniform convergence on compacta of $R$ is a consequence of the fact that every compact set can be covered by a finite number of parametric balls. Clearly

$$
F_{\Omega}\left(u_{0}\right)=\lim _{n \rightarrow \infty} E_{\Omega}\left(u_{n}\right) \leqq \lim _{n \rightarrow \infty} E\left(u_{n}\right)
$$

for every relatively compact set $\Omega$. The lemma follows as $\Omega \rightarrow R$.

6. Consider the real-valued linear operator $G_{p}(\cdot, \cdot)$ on $C \times C$ defined by

$$
G_{p}(f, g)=\int_{R \times R} g_{p}(x, y) f(x) g(y) d x d y
$$

for $f, g \in C$.

Lemma 4. If $f \in C^{\infty}$, then

$$
E\left(G_{p} f\right)=G_{p}(f, f)
$$

whenever the right-hand side is finite.

Proof. Let

$$
G_{p \Omega} f=\int_{R} g_{p \Omega}(\cdot, y) f(y) d y
$$

We have

$$
E\left(G_{p \Omega} f\right)=\int_{R \times R} g_{p \Omega}(x, y) f(x) f(y) d x d y .
$$

By the $p$-harmonicity of $G_{p} f-G_{p . \Omega} f$ on $\Omega$ and the lower semicontinuity of $E$,

$$
E\left(G_{p} f\right) \leqq \lim _{\Omega \rightarrow R} E\left(G_{p \Omega} f\right) \leqq G_{p}(|f|,|f|)<\infty .
$$

In view of Lebesgue's convergence theorem,

$$
E\left(G_{p} f\right)=\lim _{\Omega \rightarrow R} E\left(G_{p \Omega} f\right)
$$




$$
\begin{aligned}
& =\lim _{\Omega \rightarrow R} \int_{R \times R} g_{p \Omega}(x, y) f(x) f(y) d x d y \\
& =\int_{R \times R} g_{p}(x, y) f(x) f(y) d x d y \\
& =G_{p}(f, f) .
\end{aligned}
$$

\section{The $(p, q)$-biharmonic projection.}

7. As a preparation for the $(p, q)$-biharmonic projection, we introduce a number of families of functions on $R$. Let $M_{p 3}(R)$ be the class of continuous functions with finite energy integrals; $M_{p_{2}}(R)$ and $M_{p 4}(R)$ the Wiener and the Royden $p$-algebras on $R$; and $N_{p i}(R)$ the potential $p$-subalgebra of $M_{p i}(R)$ for $i=2,3,4$, (cf. [ 3 ], [10], and [11]). We shall often omit $R$ and write $M_{p i}$ and $N_{p i}$ instead of $M_{p i}(R)$ and $N_{p i}(R)$. For the sake of simplicity, we set $X_{T}=\{f \mid T f \in X\}$ and $X Y=X \cap Y$ for given classes of functions $X, Y$, and a given operator T. Furthermore, we write $M_{i j}=M_{p i}\left(M_{q j}\right)_{\Delta_{p}}$ and $N_{i j}=N_{p i}\left(N_{q j}\right)_{\Delta_{p}}$ for all $i, j$. Let $P^{\prime}, B$, and $E$ be the classes of essentially positive functions, bounded functions, and functions with finite energy integrals respectively. Set $H_{p 1}=H^{p} P^{\prime}, H_{p 2}=H^{p} B, H_{p 3}=H^{p} E$, and $H_{p 4}=H^{p} K$, where $K=B E$. It is known that the direct sum decompositions $M_{p i}=H_{p i} \oplus N_{p i}$ are valid for all $i$. The $p$-harmonic part of a function $f \in M_{p i}$ is called the $p$-harmonic projection of $M_{p i}$ and denoted by $\pi_{p i} f$. It is also known that the decompositions are orthogonal in the sense that $E(f)=E\left(\pi_{p i} f\right)+E\left(f-\pi_{p i} f\right)$ for $f \in M_{p i}$ and $i=3$, 4, (cf. e.g. [10]). Let

$$
\begin{aligned}
& F_{p 2}=\left\{f \in F_{p 1}\left|\sup _{R}\right| G_{p} f \mid<\infty\right\}, \\
& F_{p 3}=\left\{f \in F_{p_{1}} \mid G_{p}(|f|,|f|)<\infty\right\}, \\
& F_{p 4}=F_{p 2} \cap F_{p 3},
\end{aligned}
$$

and

$$
\Phi_{i j}=M_{i j}\left(F_{p i}\right)_{\left.\pi_{q j}\right\lrcorner_{p}}, \quad i, j=1,2,3,4
$$

THEOREM 1. On an arbitrary Riemannian manifold $R$ (hyperbolic if $p \equiv 0)$, the functions in $\Phi_{i j}$ have a unique decomposition into $(p, q)$ biharmonic functions and $(p, q)$-potentials:

$$
\Phi_{i j}=W_{p q} \Phi_{i j} \oplus N_{i j} \Phi_{i j}
$$

Proof. Let $f \in \Phi_{i j}$. By the decomposition theorem of $M_{p i}$ and $M_{q j}, f=\pi_{p i} f+h_{i}$ with $\pi_{p i} f \in H_{p i}$ and $h_{i} \in N_{p i}, \Delta_{p} f=\pi_{q j} \Delta_{p} f+k_{j}$ with $\pi_{q j} \Delta_{p} f \in H_{q j}$ and $k_{j} \in N_{q j}$. Since $\pi_{q j} \Delta_{p} f \in F_{p i}$ and $F_{p i} \subset F_{p 1}$, the function $w_{i j}=\pi_{p i} f+G_{p} \pi_{q j} \Delta_{p} f$ is well defined. By Lemmas 1 and 2, we see 
that $w_{i j} \in W_{p q}$ for all $i, j$, and $w_{i j} \in \Phi_{i j}$ for $i=1,2$, and all $j$. In view of Lemma 4 ,

$$
E\left(w_{i j}\right) \leqq E\left(\pi_{p i} f\right)+E\left(G_{p} \pi_{q j} \Delta_{p} f\right)=E\left(\pi_{p i} f\right)+G_{p}\left(\pi_{q j} \Delta_{p} f, \pi_{q j} \Delta_{p} f\right)<\infty
$$

for $i=3,4$. Therefore $w_{i j} \in W_{p q} \Phi_{i j}$ for all $i, j$. It remains to show that $f-w_{i j} \in N_{i j} \Phi_{i j}$. Clearly $\Delta_{p}\left(f-w_{i j}\right)=k_{j} \in N_{q j}$ and $\pi_{q j} \Delta_{p}\left(f-w_{i j}\right)=0$. By Lemma 2, $f-w_{i j}=h_{i}-G_{p} \pi_{q j} \Delta_{p} f \in N_{p i}$. Therefore $w_{i j}+\left(f-w_{i j}\right)$ is the desired decomposition.

To prove the uniqueness, let $v \in W_{p q} \Phi_{i j} \cap N_{i j} \Phi_{i j}$. Since $\Delta_{p} v \in H_{q j} \cap$ $N_{q j}=\{0\}, v \in H_{p i} \cap N_{p i}$, and consequently $v \equiv 0$ on $R$.

We call the function $w_{i j} \in W_{p q} \Phi_{i j}$ in Theorem 1 the $(p, q)$-biharmonic projection of $f \in \Phi_{i j}$. It is the solution of the $(p, q)$-biharmonic Dirichlet problem with

$$
w_{i j}\left|\beta_{i}=f\right| \beta_{i} \text { and } \Delta_{p} w_{i j}\left|\beta_{j}=\Delta_{p} f\right| \beta_{j},
$$

where $\beta_{i}$ and $\beta_{j}$ are the $p$-and $q$-harmonic boundaries corresponding to $M_{p i}$ and $M_{q j}$ respectively. From the uniqueness of the decomposition, we see that the solution is unique except for the cases $i=1$ or $j=1$. In these cases there exist singular $p$-harmonic functions which vanish on the $p$-harmonic boundary.

3. $(p, q)$-quasiharmonic classification of Riemannian manifolds.

8. The $(p, q)$-biharmonic projection was obtained in Theorem 1 for certain restricted families of functions on arbitrary Riemannian manifold. In order to relax the conditions on the families, it is necessary to impose conditions on the manifold. We shall see that such conditions are intimately related to the $(p, q)$-quasiharmonic classification of manifolds.

Denote by $O_{X}$ the class of Riemannian manifolds on which there exist no $X$-functions, and by $P$ the class of positive functions. The various $(p, q)$-quasiharmonic null-manifolds are determined completely by the $p$-harmonic Green's function and the $q$-elliptic measure:

THEOREM 2. On a q-hyperbolic Riemannian manifold $R$ (hyperbolic if $p \equiv 0)$,

(i ) $R \notin O_{Q_{p q} P}$ if and only if $G_{p} e_{q}<\infty$,

(ii) $R \notin O_{Q_{p q B} B}$ if and only if $\sup _{R} G_{p} e_{q}<\infty$,

(iii) $R \notin O_{Q_{p q} E}$ if and only if $G_{p}\left(e_{q}, e_{q}\right)<\infty$,

(iv) $R \notin O_{Q_{p q} K}$ if and only if $\sup _{R} G_{p} e_{q}<\infty$ and $G_{p}\left(e_{q}, e_{q}\right)<\infty$.

Proof. For every $u \in Q_{p q}$ and every regular subregion $\Omega \subset R$,

$$
u(x)=h_{u}^{p .}(x)+\int_{R} g_{p, 2}(x, y) e_{q}(y) d y .
$$


Suppose $R \notin O_{Q_{p q} P}$, i.e., there exists a $v \in Q_{p q} P$. Clearly $v$ is $p$-superharmonic and bounded from below on $R$. Therefore $h_{u}^{p}=\lim _{\Omega \rightarrow R} h_{u}^{p 2}$ exists. By the monotone convergence theorem,

$$
G_{p} e_{q}=\lim _{\Omega \rightarrow R} \int_{R} g_{p \Omega}(\cdot, y) e_{q}(y) d y=u-h_{u}^{p}<\infty .
$$

Conversely, $G_{p} e_{q} \in Q_{p q} P$, and (i) follows. Relation (ii) is established in a similar manner.

Suppose $R \notin O_{Q_{p q} E}$ and take a $v \in Q_{p q} E$. For every regular subregion $\Omega \subset R$,

$$
v=h_{v}^{p Q}+G_{p \Omega} e_{q}
$$

and

$$
E(v)=E\left(h_{v}^{p . Q}\right)+E\left(G_{p g} e_{q}\right) .
$$

As in the proof of Lemma 4,

$$
E\left(G_{p, \Omega} e_{q}\right)=G_{p, \Omega}\left(e_{q}, e_{q}\right) .
$$

The monotone convergence theorem yields

$$
\lim _{\Omega \rightarrow R} G_{p, \Omega}\left(e_{q}, e_{q}\right)=G_{p}\left(e_{q}, e_{q}\right) \text {. }
$$

Since $G_{p}\left(e_{q}\right)-G_{p \Omega}\left(e_{q}\right)$ is $p$-harmonic on $\Omega$, Lemma 2 implies

$$
E\left(G_{p} e_{q}\right) \leqq \lim _{\Omega \rightarrow R} E\left(G_{p \Omega} e_{q}\right) \leqq E(v)<\infty .
$$

By Lebesgue's convergence theorem,

$$
G_{p}\left(e_{q}, e_{q}\right)=E\left(G_{p} e_{q}\right)<\infty .
$$

Conversely, if $G_{p}\left(e_{q}, e_{q}\right)<\infty$, then $G_{p} e_{q}<\infty$ and $\Delta_{p} G_{p} e_{q}=e_{q}$. By virtue of

$$
E\left(G_{p} e_{q}\right) \leqq \lim _{2 \rightarrow R} E\left(G_{p .2} e_{q}\right) \leqq G_{p}\left(e_{q}, e_{q}\right)<\infty,
$$

$G_{p} e_{q} \in Q_{p q} E$ and (iii) follows. The last assertion of Theorem 2 is an immediate consequence of (ii) and (iii).

9. An important bi-product of the proof of Theorem 1 is that the $(p, q)$-biharmonic functions restricted to the class $\Phi_{i j}$ can be uniquely decomposed into the $p$-harmonic part and the potential part:

THEOREM 3. On an arbitrary Riemannian manifold $R$ (hyperbolic if $p \equiv 0$ ), every function $w_{i j} \in W_{p q} \Phi_{i j}$ can be uniquely written as

$$
w_{i j}=u_{i}+G_{p} v_{j},
$$


with $u_{i} \in H_{p i}$ and $v_{j} \in H_{q j}$ for $i, j=1,2,3,4$.

\section{Nondegenerate manifolds.}

10. We shall show that, by imposing a suitable condition on the manifold $R$, the restrictions we have set on the functions which have $(p, q)$-biharmonic projections can be relaxed.

We write $X_{1}=P, X_{2}=B, X_{3}=E, X_{4}=K$, and we let $W_{p q} X_{1}\left(X_{j}\right)_{A_{p}}$ stand for $W_{p q} M_{p 1}\left(X_{j}\right)_{\Delta_{p}}$.

Theorem 4. On a Riemannian manifold which carries $Q_{p q} X_{i^{-}}$ functions,

$$
M_{i j}=W_{p q} X_{i}\left(X_{j}\right)_{\lrcorner_{p}} \oplus N_{i j}
$$

with $i=1,2,3,4$, and $j=2,4$.

Proof. It is sufficient to show that $f \in W_{p q} B_{\Delta_{p}}$ implies the $p$ harmonizability of $f$ on $R \notin O_{Q_{p q} P}$.

For every regular subregion $Q$ of $R$, and every $f \in W_{p q} B_{\Delta_{p}}$,

$$
f=h_{f}^{p \Omega}+\int_{R} g_{p \Omega}(\cdot, y) \Delta_{p} f(y) d y .
$$

Since $R \notin O_{Q_{p q} P}, G_{p} e_{q}<\infty$ by Theorem 2. In view of $\left|g_{p \rho} \cdot \Delta_{p} f\right| \leqq k \cdot g_{p} e_{q}$ for some constant $k$, the Lebesgue convergence theorem implies the existence of the limit of $\lim _{\Omega \rightarrow R} \int_{R} g_{p \Omega}(\cdot, y) \Delta_{p} f(y) d y$. Thus $h_{f}^{p \rho}$ converges, and $f$ is $p$-harmonizable.

11. With suitable conditions imposed on the manifold, we have the following direct sum decompositions of $(p, q)$-biharmonic functions:

THEOREM 5. On a Riemannian manifold $R$ which carries positive $Q_{p q}$-functions,

$$
W_{p q} X_{i}\left(X_{j}\right)_{\lrcorner_{p}} \subset H_{p i} \oplus G_{p} H_{q j}
$$

with $i=1,2,3,4$, and $j=2,4$. Moreover,

$$
W_{p q} X_{i}\left(X_{j}\right)_{\Lambda_{p}}=H_{p i} \oplus G_{p} H_{q j}
$$

if and only if $R \notin O_{Q_{p q}} X_{i}$.

The proof makes use of Theorems 2,3 , and 4 .

On a manifold $R \notin O_{Q_{p q} x}$, let $\varphi, \psi$ be continuous functions on the harmonic boundaries $\beta_{i}$ and $\beta_{j}$ corresponding to $M_{p i}$ and $M_{q j}$ respec- 
tively. The second assertion of Theorem 5 implies that if $h_{\varphi}^{p}$ and $h_{\varphi}^{q}$ are solutions of the $p$-and $q$-harmonic boundary value problems with boundary values $\phi$ and $\psi$ respectively, then our $(p, q)$-biharmonic Dirichlet problem has a solution which is in $W_{p q} X_{i}\left(X_{j}\right)_{A_{p}}$ and takes the form $h_{\varphi}^{p}+G_{p} h_{\varphi}^{q}$.

\section{Special density functions.}

12. In the case that the density function is bounded from below by a positive constant, we have more explicit results:

THEOREM 6. If $\inf _{R} p(x)>0$ on a q-hyperbolic Riemannian manifold, then

$$
M_{i j}=W_{p q} X_{i}\left(X_{j}\right)_{\lrcorner_{p}} \oplus N_{i j}
$$

and

$$
W_{p q} X_{i}\left(X_{j}\right)_{\lrcorner_{p}}=H_{p i} \oplus G_{p} H_{q j}
$$

with $i=1,2$, and $j=2,4$. Furthermore, if $\int_{R} p(x) d x<\infty$, then the above assertion is true also for $i=3,4$.

Proof. To prove the first assertion, it is sufficient to show that $R \notin O_{Q_{p q} B}$ for $\inf _{R} p(x)>0$. On every regular subregion $\Omega$, we have $1=h_{1}^{p \Omega}+\int_{R} g_{p \Omega}(\cdot, y) p(y) d y$, and consequently $G_{p} p \leqq 1$ upon letting $\Omega \rightarrow R$. Therefore $G_{p} e_{q} \leqq G_{p} 1 \leqq 1 / m$ with $m=\inf _{R} p$. By Theorem 2 , $R \notin O_{Q_{p q} B}$. Suppose furthermore that $\int_{R} p(x) d x<\infty$. Then the volume of $R$ is $V(R)=\int_{R} d x \leqq 1 / m \int_{R} p(x) d x \stackrel{\int_{R}}{<} \infty$ and

$$
G_{p}\left(e_{q}, e_{q}\right) \leqq \frac{1}{m^{2}} V(R)<\infty \text {. }
$$

The second assertion follows from Theorem 2.

13. By the fact that $g_{p}(x, y) \leqq g_{r}(x, y)$ for $p \geqq r$, and Theorem 2 , we have the following:

Proposition. On a q-hyperbolic Riemannian manifold $R$ (hyperbolic if $p \equiv 0$ ),

(i) $O_{Q_{p q} P} \subset O_{Q_{p q} B} \subset O_{Q_{p q} K}$, and $O_{Q_{p q} P} \subset O_{Q_{p q} E} \subset O_{Q_{p q} K}$,

(ii) $O_{Q_{p q} X} \subset O_{Q_{r q} X}$ for $p \geqq r$,

(iii) $O_{Q_{p q} X} \subset O_{Q_{p s} X}$ for $q \geqq s$,

(iv) $O_{Q_{p q} X} \subset O_{Q_{r s} X}$ for $p \geqq r$ and $q \geqq s$, with $X=P, B, E$ or $K$. 
We note that if $R$ is $q$-parabolic, $Q_{p q}=H^{p}$ and $\varnothing=O_{H^{p_{P}}} \subset O_{H^{p_{B}}} \subset$ $O_{H^{p}}=O_{H^{p} K}$, that is, (i) is still true. However, (ii)-(iv) are no longer valid, for $\varnothing=O_{H^{p} P}=O_{H^{r} P}, O_{H^{r} r_{B}} \subset O_{H^{p} p_{B}}$, and $O_{H^{r} r_{E}}=O_{H^{r} K} \subset O_{H^{p}}=$ $O_{H^{p}}$ if $p \geqq r$.

From (iv) of the above proposition, we see that if the $(r, s)$ biharmonic Dirichlet problem is solvable by the decomposition method of Theorem 5, then the $(p, q)$-biharmonic boundary value problem has a solution for $p \geqq r$ and $q \geqq s$. In particular, the $(p, q)$-biharmonic Dirichlet problem is solvable if the biharmonic problem is.

\section{REFERENCES}

1. C. Constantinescu and A. Cornea, Ideale Ränder Riemannscher Flächen, Springer, 1963, 244.

2. P. R. Garabedian, Partial Differential Equations, Wiley, New York, 1964, 672.

3. Y. K. Kwon, L. Sario and J. Schiff, Bounded energy-finite solutions of $\Delta u=P u$ on a Riemannian manifold, Nagoya Math. J., 42 (1971), 95-108.

4. Y. K. Kwon, L. Sario and B. Walsh, Behavior of biharmonic functions on Wiener's and Royden's compactifications, Ann. Inst. Fourier (Grenoble), 21 (1971), 217-226.

5. M. Nakai and L. Sario, Biharmonic classification of Riemannian manifolds, Bull. Amer. Math. Soc., 77 (1971), 432-436.

6. Quasiharmonic classification of Riemannian manifolds, Proc. Amer. Math. Soc., 31 (1972), 165-169.

7. - A property of biharmonic functions with Dirichlet finite Laplacians, Math. Scand., (to appear).

8. - Dirichlet finite biharmonic functions with Dirichlet finite Laplacians, Math. Z., 122 (1971), 203-216.

9. H. L. Royden, The equation $\Delta u=p u$ and the classification of open Riemann surfaces, Ann. Acad. Sci. Fenn., A. I., 271 (1959).

10. L. Sario and M. Nakai, Classification Theory of Riemann Surfaces, Springer, 1970, 446.

11. L. Sario, C. Wang and M. Range, Biharmonic projection and decomposition, Ann. Acad. Sci. Fenn., A.I., 494 (1971).

12. H. Tanaka, On Wiener functions of order $m$, (to appear).

Received March 29, 1971. The work was sponsored by the U. S. Army Research Office-Durham, Grant DA-ARO-D-31-124-71-G20, University of California, Los Angeles. 


\title{
PACIFIC JOURNAL OF MATHEMATICS
}

\author{
EDITORS
}

H. SAMELSON

Stanford University

Stanford, California 94305

C. R. HOBBY

University of Washington

Seattle, Washington 98105

\section{J. DugundJI}

Department of Mathematics

University of Southern California

Los Angeles, California 90007

RICHARD ARENS

University of California

Los Angeles, California 90024

\section{ASSOCIATE EDITORS}
E. F. BeCKeNBACH
B. H. NeUmanN
F. WoLF
K. YoSHIDA

\section{SUPPORTING INSTITUTIONS}

\author{
UNIVERSITY OF BRITISH COLUMBIA \\ CALIFORNIA INSTITUTE OF TECHNOLOGY \\ UNIVERSITY OF CALIFORNIA \\ MONTANA STATE UNIVERSITY \\ UNIVERSITY OF NEVADA \\ NEW MEXICO STATE UNIVERSITY \\ OREGON STATE UNIVERSITY \\ UNIVERSITY OF OREGON \\ OSAKA UNIVERSITY
}

\author{
UNIVERSITY OF SOUTHERN CALIFORNIA \\ STANFORD UNIVERSITY \\ UNIVERSITY OF TOKYO \\ UNIVERSITY OF UTAH \\ WASHINGTON STATE UNIVERSITY \\ UNIVERSITY OF WASHINGTON

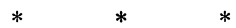 \\ AMERICAN MATHEMATICAL SOCIETY \\ NAVAL WEAPONS CENTER
}

The Supporting Institutions listed above contribute to the cost of publication of this Journal, but they are not owners or publishers and have no responsibility for its content or policies.

Mathematical papers intended for publication in the Pacific Journal of Mathematics should be in typed form or offset-reproduced, (not dittoed), double spaced with large margins. Underline Greek letters in red, German in green, and script in blue. The first paragraph or two must be capable of being used separately as a synopsis of the entire paper. The editorial "we" must not be used in the synopsis, and items of the bibliography should not be cited there unless absolutely necessary, in which case they must be identified by author and Journal, rather than by item number. Manuscripts, in duplicate if possible, may be sent to any one of the four editors. Please classify according to the scheme of Math. Rev. Index to Vol. 39. All other communications to the editors should be addressed to the managing editor, Richard Arens, University of California, Los Angeles, California, 90024.

50 reprints are provided free for each article; additional copies may be obtained at cost in multiples of 50 .

The Pacific Journal of Mathematics is published monthly. Effective with Volume 16 the price per volume (3 numbers) is $\$ 8.00$; single issues, $\$ 3.00$. Special price for current issues to individual faculty members of supporting institutions and to individual members of the American Mathematical Society: $\$ 4.00$ per volume; single issues $\$ 1.50$. Back numbers are available.

Subscriptions, orders for back numbers, and changes of address should be sent to Pacific Journal of Mathematics, 103 Highland Boulevard, Berkeley, California, 94708.

PUBLISHED BY PACIFIC JOURNAL OF MATHEMATICS, A NON-PROFIT CORPORATION

Printed at Kokusai Bunken Insatsusha (International Academic Printing Co., Ltd.), 270, 3-chome Totsuka-cho, Shinjuku-ku, Tokyo 160, Japan. 


\section{Pacific Journal of Mathematics}

\section{Vol. 41, No. $3 \quad$ BadMonth, 1972}

George E. Andrews, Two theorems of Gauss and allied identities proved arithmetically.................................. 563

Stefan Bergman, On pseudo-conformal mappings of circular domains . . . . 579

Beverly L. Brechner, On the non-monotony of dimension ............ 587

Richard Anthony Brualdi and John H. Mason, Transversal matroids and Hall's theorem .................................... 601

Philip Throop Church and James Timourian, Differentiable maps with

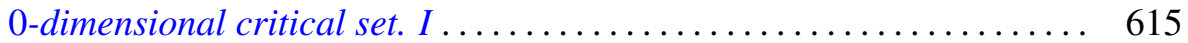

John H. E. Cohn, Squares in some recurrent sequences ............. 631

Robert S. Cunningham, Edgar Andrews Rutter and Darrell R. Turnidge, Rings of quotients of endomorphism rings of projective modules ......

Eldon Dyer and S. Eilenberg, An adjunction theorem for locally equiconnected spaces................................... 669

Michael W. Evans, On commutative P. P. rings................. 687

Ronald Lewis Graham, Hans Sylvain Witsenhausen and Hans Zassenhaus, On tightest packings in the Minkowski plane ..................

Stanley P. Gudder, Partial algebraic structures associated with

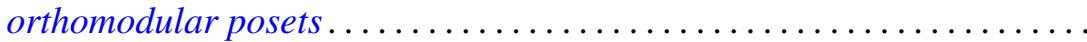

Karl Edwin Gustafson and Gunter Lumer, Multiplicative perturbation of

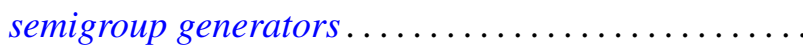

Kurt Kreith and Curtis Clyde Travis, Jr., Oscillation criteria for selfadjoint elliptic equations...

Lawrence Louis Larmore, Twisted cohomology theories and the single obstruction to lifting...........................

Jorge Martinez, Tensor products of partially ordered groups . . .

Robert Alan Morris, The inflation-restriction theorem for Amitsur cohomology ...

Leo Sario and Cecilia Wang, The class of $(p, q)$-biharmonic functions ...

Manda Butchi Suryanarayana, On multidimensional integral equations of

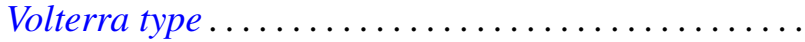

